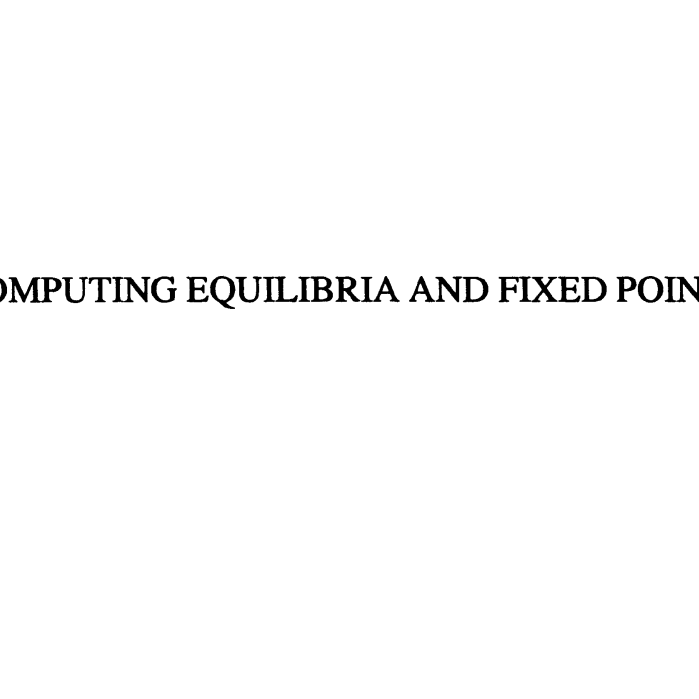


THEORY AND DECISION LIBRARY

General Editors: W. Leinfellner (Vienna) and G. Eberlein (Munich)

Series A: Philosophy and Methodology of the Social Sciences

Series B: Mathematical and Statistical Methods

Series C: Game Theory, Mathematical Programming and Operations Research

\section{SERIES C: GAME THEORY, MATHEMATICAL PROGRAMMING AND OPERATIONS RESEARCH}

\section{VOLUME 21}

Editor: S. H. Tijs (University of Tilburg); Editorial Board: E.E.C. van Damme (Tilburg), H. Keiding (Copenhagen), J.-F. Mertens (Louvain-la-Neuve), H. Moulin (Durham), S. Muto (Tokyo University), T. Parthasarathy (New Delhi), B. Peleg (Jerusalem), H. Peters (Maastricht), T. E. S. Raghavan (Chicago), J. Rosenmüller (Bielefeld), A. Roth (Pittsburgh), D. Schmeidler (Tel-Aviv), R. Selten (Bonn), W. Thomson (Rochester, NY).

Scope: Particular attention is paid in this series to game theory and operations research, their formal aspects and their applications to economic, political and social sciences as well as to socio-biology. It will encourage high standards in the application of game-theoretical methods to individual and social decision making. 


\title{
COMPUTING EQUILIBRIA AND FIXED POINTS
}

\section{The Solution of Nonlinear Inequalities}

\author{
by \\ ZAIFU YANG \\ Yokohama National University, Japan
}

SPRINGER-SCIENCE+BUSINESS MEDIA, B.V. 
A C.I.P. Catalogue record for this book is available from the Library of Congress.

Softcover reprint of the hardcover 1st edition 1999

No part of the material protected by this copyright notice may be reproduced or utilized in any form or by any means, electronic or mechanical, including photocopying, recording or by any information storage and retrieval system, without written permission from the copyright owner 
To the memory of my grandparents 


\section{Table of Contents}

Preface v v

1 Mathematical Preliminaries 1

1.1 Introduction . . . . . . . . . . . . . 1

1.2 Basic notation and terminology $\ldots \ldots \ldots \ldots$

1.3 Basic polyhedral theory . . . . . . . . . . . 5

1.4 Simplices, triangulations and graphs . . . . . . . 8

1.5 Brouwer's theorem and Sperner's lemma . . . . . . . . . 17

1.6 Kakutani's theorem and Browder's theorem . . . . . . . 25

1.7 Theorems of Tarski, Caristi and Ekeland . . . . . . . . . 31

2 Applications in Game Theory and Economics 37

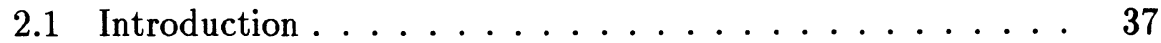

2.2 The core of a game ................. 37

2.3 Nash, perfect Nash, and proper Nash equilibria . . . . . 40

2.4 A pure exchange economy . . . . . . . . . . 43

2.5 An exchange economy with price rigidities . . . . . . 46

2.6 An exchange economy with linear technologies ...... 51

2.7 An exchange economy with non-convex technologies $\ldots . .53$

2.8 An exchange economy with indivisibilities . . . . . . 55

2.9 An exchange economy under uncertainty . . . . . . . 57

3 First Algorithms for Approximating Fixed Points 61

3.1 Introduction . . . . . . . . . . . . 61

3.2 Integer labeling and approximation $\ldots \ldots \ldots \ldots 6$

3.3 Scarf's algorithm . . . . . . . . . . . . . . 65

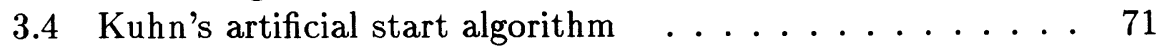

3.5 Kuhn's variable dimension algorithm . . . . . . . . 74

3.6 Concluding remarks . . . . . . . . . . . . 77

4 Simplicial Homotopy Algorithms 79

4.1 Introduction . . . . . . . . . . . . . . . 79

4.2 Vector labeling and lexicographic system ... . . . 80

4.3 Merrill's algorithm . . . . . . . . . . . . . . 84

4.4 Eaves' algorithm on $S^{n} \times[1, \infty) \ldots \ldots \ldots 90 \ldots$

4.5 Eaves-Saigal's algorithm on $\mathbf{R}^{n} \times[1, \infty) \ldots \ldots \ldots$ 
5 Variable Dimension Restart Algorithms 101

5.1 Introduction . . . . . . . . . . . . . . . . 101

5.2 Van der Laan-Talman's algorithm on $S^{n} \ldots \ldots \ldots \ldots 10 . \ldots 1$

5.3 Van der Laan-Talman's algorithm on $\mathbb{R}^{n} \ldots \ldots \ldots . \ldots 105$

5.4 Concluding remarks . . . . . . . . . . . . . 112

6 An Algorithm for Integer Linear Programming 115

6.1 Introduction . . . . . . . . . . . . . . . 115

6.2 The problem: an NP-complete problem . . . . . . . 116

6.3 The integer labeling algorithm . . . . . . . . . 120

6.4 Unimodular transformation . . . . . . . . . . . . . 134

6.5 Numerical results . . . . . . . . . . . . . . . . . 142

7 Refinement and Stability of Stationary Points 147

7.1 Introduction . . . . . . . . . . . . . . . 147

7.2 The concept of robust stationary point . . . . . . 149

7.3 The $P$-triangulation of the unit simplex $\ldots \ldots \ldots \ldots$

7.4 The adaptive simplicial algorithm . . . . . . . . . 154

7.5 Examples and resolution of degeneracy . . . . . . 162

7.6 Extension to general polytopes . . . . . . . . . 167

8 Computing a Continuum of Zero Points 171

8.1 Introduction . . . . . . . . . . . . 171

8.2 A general problem and an economic model . . . . . . . 173

8.3 An algorithm for computing a continuum of zero points . . 174

8.4 Existence of a continuum of zero points . . . . . . . 183

8.5 An illustration of the algorithm . . . . . . . . . 187

8.6 A constructive proof for Browder's theorem . . . . . . . 191

9 Computing Stationary Points on Polytopes 195

9.1 Introduction . . . . . . . . . . . . . . 195

9.2 Basic theorems for resolving degeneracy $\ldots \ldots \ldots \ldots$

9.3 An algorithm for finding zero points . . . . . . . . 204

9.4 An algorithm for finding stationary points . . . . . . 210

9.5 The $V$-triangulation of polytopes . . . . . . . . 214

10 The Computation of Antipodal Fixed Points 217

10.1 Introduction . . . . . . . . . . . . . 217

10.2 Borsuk-Ulam's theorem and Tucker's theorem $\ldots \ldots . . .217$

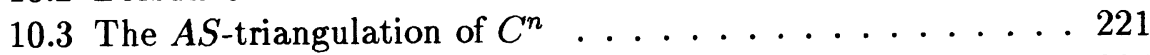

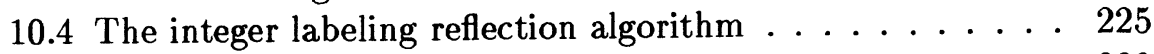

10.5 The vector labeling reflection algorithm . . . . . . . 229 
11 Computing All Roots of Univariate Polynomials 239

11.1 Introduction . . . . . . . . . . . . . . 239

11.2 An integer labeling rule . . . . . . . . . . . . 240

11.3 A triangulation of $\mathbb{C} \times[-1, \infty) \ldots \ldots \ldots \ldots \ldots$

11.4 Kuhn's algorithm . . . . . . . . . . . . 245

11.5 Convergence proofs . . . . . . . . . . . . 250

11.6 Complexity analysis and numerical examples . . . . . . 260

12 Gröbner Bases for Solving Polynomial Equations 265

12.1 Introduction . . . . . . . . . . . . . 265

12.2 Basic concepts and Hilbert's basis theorem . . . . . 266

12.3 Multi-variable division algorithm . . . . . . . . . 269

12.4 Gröbner bases . . . . . . . . . . . . . 273

12.5 Buchberger's algorithm . . . . . . . . . . . 275

12.6 Hilbert's zero point theorem . . . . . . . . . . . . 282

12.7 Applications: the complementarity problems . . . . . . 286

13 Intersection Theory 289

13.1 Introduction . . . . . . . . . . . . . . . 289

13.2 A general intersection theorem . . . . . . . . 290

13.3 Intersection theorems on the unit simplex . . . . . . . 293

13.4 Intersection theorems on polytopes . . . . . . . . 296

13.5 Theorems with multiple intersection points . . . . . . . 301

13.6 An m.p.b. intersection theorem . . . . . . . . . . 302

13.7 Helly's intersection theorem . . . . . . . . . . . 308

14 Sperner Theory $\quad 311$

14.1 Introduction . . . . . . . . . . . . . . 311

14.2 Preliminaries for analysis . . . . . . . . . . . 312

14.3 Main integer labeling theorems . . . . . . . . . . . 313

14.4 Applications to the unit simplex . . . . . . . . 317

14.5 Applications to polytopes . . . . . . . . . . 320

$\begin{array}{ll}\text { References } & \mathbf{3 2 3}\end{array}$

$\begin{array}{ll}\text { Index } & \mathbf{3 3 5}\end{array}$ 


\section{Preface}

This book is an introduction to the computation of equilibria, fixed points and stationary points in finite dimension spaces. We wrote this book with three goals in mind:

(i) To give a comprehensive introduction to fixed point methods and to the definition and construction of Gröbner bases;

(ii) To discuss several interesting applications of these methods in the fields of general equilibrium theory, game theory, mathematical programming, algebra and symbolic computation;

(iii) To introduce several advanced fixed point and stationary point theorems.

These methods and topics are of interest not only to economists and game theorists concerned with the computation and existence of equilibrium outcomes in economic models and cooperative and non-cooperative games, but also to applied mathematicians, computer scientists and engineers dealing with models of highly nonlinear systems of equations (or polynomial equations). Many of the results in this book are quite new and only available in research papers. We have also made the mathematical prerequisites to the book very modest so that it should be suitable for an advanced undergraduate or a first year graduate student.

For a long time the arduous problem of solving a system of nonlinear equations or inequalities has been a challenge. In fact, this problem dates back to the beginning of human civilization when the ancient Babylonians, Chinese, Egyptians and Greeks were studying specific examples of linear and quadratic equations with very few variables (see Midonick [1968] and van der Waerden [1983]). However, it was not until the appearance of Hilbert's Nullstellensatz (Hilbert's zero point theorem) in the late nineteenth century, and Brouwer's fixed point theorem in the early twentieth century, that a breakthrough was made in quest of the existence of a solution to a general system of nonlinear (polynomial) equations. Generally speaking, Hilbert's zero point theorem gives a sufficient and necessary condition for the existence of a solution to a system of nonlinear polynomial equations. Furthermore Brouwer's fixed point theorem states that every continuous function from a nonempty, convex and compact subset of the Euclidean space into itself has at least one fixed point, i.e., a point in the set being mapped into itself. For many years these theorems and related results 
appeared to be remote from any practical use because of their mathematical abstractness and the non-constructive nature of their proofs. However, in the 1960s, Scarf developed a finite algorithm for finding an approximate fixed point of a continuous function from the unit simplex into itself. This algorithm provided the first constructive proof to Brouwer's fixed point theorem. At the same time, Buchberger discovered a finite algorithm for manipulating systems of nonlinear polynomial equations, which can actually determine whether a system of polynomial equations has a solution or not, (though it does not show how to find a solution); this gave the first constructive proof to Hilbert's zero point theorem. The former algorithm laid the foundation for fixed point methods; the latter one, the foundation for Gröbner basis methods. These two pioneering studies have stimulated many researchers to extend the applicability and improve the efficiency of these methods. More specifically, efficient fixed point algorithms have been designed for applications in general equilibrium theory, game theory, mathematical programming and engineering problems, whereas Gröbner basis algorithms have found applications in computational algebra, symbolic computation and computer science. This book is intended to introduce the recent fixed point algorithms, the basic Gröbner basis algorithm, and their applications, to a wider audience.

Note that the aim of this book is the solution of highly nonlinear systems of equations which are required only to be continuous, or upper semicontinuous. Therefore, the book does not deal with conceptually simpler methods to solve smooth systems that require first or even higher order derivatives, like Newton methods and continuation methods. The interested reader is referred to Ortega and Rheinbolt [1970], Kellogg, Li and Yorke [1976], Smale [1976], Allgower and Georg [1990], Harker and Pang [1990], Yu and Lin [1996].

The book is organized as follows. Chapter 1 describes the mathematical tools needed to understand the rest of the book. It includes basic notation and concepts which will be used throughout the book, as well as several classic theorems from polyhedral theory, definitions of graphs, simplices, and triangulations. We also present Brouwer's fixed point theorem, Kakutani's fixed point theorem, Browder's fixed point theorem, KnasterKuratowski-Mazurkiewicz's (KKM) lemma and Sperner's lemma which will be demonstrated in constructive manners in the following chapters. Furthermore, some other types of fixed point theorems such as Tarski's theorem, Caristi's theorem, and Ekeland's theorem are introduced and proved.

It is a rather common belief that the ultimate goal of even the most abstract and theoretical research work should be its practical applicability. Therefore, Chapter 2 discusses several applications of fixed point theorems. In fact, the topics under consideration have been the major source of 
motivation and inspiration for the development of fixed point algorithms. These topics include the core of cooperative games, the Nash, perfect Nash and proper Nash equilibria of non-cooperative finite games, the Walrasian equilibrium of a pure exchange economy, the Drèze equilibrium and supplyconstrained (or unemployment) equilibrium of a general exchange economy under price rigidities, the equilibrium of an exchange economy with increasing returns to scale, the competitive equilibrium of an exchange economy with indivisibilities and money, and the Radner equilibrium of an exchange economy under uncertainty.

In Chapters 3, 4 and 5 we systematically introduce the classic fixed point algorithms as developed over the last thirty years and familiarize the reader with the basic theory and techniques of these algorithms. These algorithms will be presented approximately in the order of the events. In doing so, we hope that the reader will easily see how these methods were developed and improved, and where there is room for further improvement. We discuss these methods in an accessible way but with no loss of mathematical rigor. More precisely, these algorithms include the first algorithm of Scarf based on primitive sets, the algorithms of Kuhn based on simplices, Merrill's (and Kuhn-MacKinnon's) restart algorithm, the homotopy algorithms of Eaves, and Eaves-Saigal, and the variable dimension restart algorithms of van der Laan-Talman.

Chapter 6 presents an algorithm for solving a class of integer programming problems which are known to be intractable, as they cannot be shown to be solvable in polynomial time. The algorithm is based on both a specific integer labeling rule and a triangulation of the Euclidean space. Starting with an arbitrarily chosen integer vector, the algorithm terminates within a finite number of steps either yielding an optimal solution or proving that the problem has no feasible integer solution. Prominent features of the algorithm are its computational simplicity, flexibility and stability. Numerical results are also reported.

In Chapter 7 the stability and refinement of stationary points is studied. There is growing evidence that a continuous function may have many stationary points and some of them are undesirable with regard to stability. Hence, it is very important to eliminate these stationary points. To achieve this goal, we introduce a new solution to the stationary point problem. This solution has certain stable properties and is called a robust stationary point. The concept of robust stationary point is a refinement of that of stationary point. Although a stationary point need not be robust, it has been shown that every continuous function on polytopes has at least one robust stationary point. A simplicial algorithm is developed to compute such a robust stationary point. An important application of the algorithm is the computation of proper Nash equilibria and robust Walrasian equi- 
libria. This theory provides a unifying framework for studying the stability and refinement of Walrasian equilibria and Nash equilibria.

Chapter 8 introduces a new fixed point theorem which states that under some mild conditions there exists a continuum of fixed points for a pointto-set mapping. Although many fixed point theorems have been established following the appearance of Brouwer's fixed point theorem, to date the existence of results for multiple fixed points is very rare. This theorem is proved in a constructive manner by means of a simplicial algorithm. Applying the algorithm to the model of an exchange economy under price rigidities, it is proved that there exists a connected set of constrained equilibria linking together two trivial no-trade equilibria. The algorithm is also applied so as to provide a first constructive proof for Browder's fixed point theorem. This chapter is based on the work of Herings, Talman and Yang [1996].

In Chapter 9, two algorithms are discussed for computing zero points, respectively, stationary points of a continuous function on a general polytope. These methods were first developed by Talman and Yamamoto [1989] and later modified by Fujishige and Yang [1998]. Talman and Yamamoto's method is the first to compute a stationary point of any continuous function on a general polytope. In simplicial algorithms we often have to resolve degeneracy problems. Here, a general method is presented for dealing with these problems. Moreover, a new existence theorem of zero points on general polytopes is introduced which generalizes the well-known multivariate mean value theorem.

In Chapter 10 an algorithm is presented to compute an antipodal fixed point of a continuous function on a symmetric cubical set. As a result, constructive proofs of the well-known Borsuk-Ulam's fixed point theorem and Tucker's combinatorial theorem are obtained. This algorithm is designed to find a simplex in a given triangulation, which contains an approximate antipodal fixed point. Furthermore, we present a generalization of BorsukUlam's theorem to a point-to-set mapping. This chapter largely reflects the work of Todd and Wright [1980], Freund and Todd [1981], and van der Laan [1984].

In Chapter 11, we discuss Kuhn's algorithm for computing all roots of a univariate polynomial with complex coefficients. Consequently, we also obtain a constructive proof of the Fundamental Theorem of Algebra. Kuhn's algorithm is the first to compute all roots of a univariate polynomial. The algorithm is built upon both a particular integer labeling rule and a triangulation of the complex plane. The complexity of the algorithm is analyzed and numerical results are reported.

In Chapter 12, we provide the definition of a Gröbner basis and then show how to compute it by using Buchberger's algorithm. Then, we proceed to discuss the famous Hilbert's basis theorem and Hilbert's zero point 
theorem and solve the ideal membership problem in a constructive manner. Furthermore, we give a method to solve systems of multivariate polynomial equations with complex coefficients. To do so, we first determine whether such a system even has solutions. If a solution exists, we determine whether it has a finite number of solutions. If the system has a finite number of solutions, then all solutions in the system can be found by using Kuhn's algorithm successively. If the system has an infinite number of solutions, we can apply a homotopy algorithm to find as many solutions as we wish to have. Thus, this procedure for solving a system of polynomial equations is a remarkable extension of Gauss-Jordan elimination method for solving a system of linear equations. Meanwhile, we also discuss how to apply the above theories to solve a large class of linear and nonlinear complementarity problems.

Finally, in Chapters 13 and 14 we discuss both the theory on intersection points and Sperner's theory. These theories are closely related to fixed point theory and provide geometric intuitions to fixed point algorithms. In particular, three unifying combinatorial theorems on general polytopes are presented. The first is a general intersection theorem, while the second and third are two general integer labeling theorems. Many well-known results, such as the lemmas of Sperner, KKM, Scarf, Shapley, among others, will be derived from these three general theorems. We also discuss intersection theorems of Freidenfelds and Herings-Talman which state conditions for the existence of a continuum of intersection points. Furthermore, we present several permutation-based generalizations of Brouwer's fixed point theorem, KKM lemma and Sperner's lemma. By applying such generalization we prove the existence of an equilibrium in an economy with multiple indivisibilities and money.

\section{Acknowledgments}

I would like to thank the many people who have helped me write this book. To the aforementioned individuals who created this exciting research field, my gratitude is obvious. To my teachers, friends and co-researchers, Dolf Talman, Gerard van der Laan, Yoshi Yamamoto, Kai-zhou Chen, I have come to incur an incalculable intellectual debt. I cannot find the words to pay adequate tribute to them. Over the years, I have had many interesting and stimulating discussions with my friends Antoon van den Elzen and Jean-Jacques Herings to whom I owe a great deal. I am also indebted to Walter Forster, Satoru Fujishige, Pieter Ruys, Stef Tijs, Ze-ke Wang, and Xinhui Xing for their encouragement and help. I would also like to 
express my gratitude to my colleagues at Tilburg, Tsukuba and Yokohama for creating stimulating research environments and offering me the opportunity to complete this work. I am also grateful to Allard Winterink and Siep Kroonenberg of Kluwer Academic Publishers for Allard's advice and for Siep's technical assistance. Finally, I send my very special thanks to my wife and my parents for their understanding and support. 